\title{
Classification of Ipsilateral Breast Tumor Recurrences After Breast Conservation Therapy Can Predict Patient Prognosis and Facilitate Treatment Planning
}

\author{
Min Yi, MD*, Thomas A. Buchholz, MD†, Funda Meric-Bernstam, MD*, Isabelle Bedrosian, MD*, \\ Rosa F. Hwang, MD*, Merrick I. Ross, MD*, Henry M. Kuerer, MD, PhD*, Sheng Luo, PhD \\ Ana M. Gonzalez-Angulo, MD§, Aman U. Buzdar, MD§, W. Fraser Symmans, MDף, Barry W. Feig, MD*, \\ Anthony Lucci, MD*, Eugene H. Huang, MD\|, and Kelly K. Hunt, MD*
}

\begin{abstract}
Objective: To classify ipsilateral breast tumor recurrences (IBTR) as either new primary tumors (NP) or true local recurrence (TR). We utilized 2 different methods and compared sensitivities and specificities between them. Our goal was to determine whether distinguishing NP from TR had prognostic value.

Background: After breast-conservation therapy, IBTR may be classified into 2 distinct types (NP and TR). Studies have attempted to classify IBTR by using tumor location, histologic subtype, DNA flow cytometry data, or geneexpression profiling data.

Methods: A total of $447(7.9 \%)$ of 5660 patients undergoing breastconservation therapy from 1970 to 2005 experienced IBTR. Clinical data from 397 patients were available for review. We classified IBTRs as NP or TR on the basis of either tumor location and histologic subtype (method 1) or tumor location, histologic subtype, estrogen receptor status and human epidermal growth factor receptor 2 status (method 2). Kaplan-Meier curves and log-rank tests were used to evaluate overall and disease-specific survival differences between the 2 groups. Classification methods were validated by calculating sensitivity and specificity values using a Bayesian method.

Results: Of 397 patients, 196 (49.4\%) were classified as NP by method 1 and $212(53.4 \%)$ were classified as NP by method 2 . The sensitivity and specificity values were 0.812 and 0.867 for method 1 and 0.870 and 0.800 for method 2 , respectively. Regardless of method used, patients classified as NP developed contralateral breast carcinoma more often but had better 10 -year overall and disease-specific survival rates than those classified as TR. Patients with TR were more likely to develop metastatic disease after IBTR

Conclusion: Ipsilateral breast tumor recurrences classified as TR and NP had clinically different features, suggesting that classifying IBTR may provide clinically significant data for the management of IBTR.
\end{abstract}

From the * Departments of Surgical Oncology, $†$ Radiation Oncology, §Breast Medical Oncology, and 9 Pathology, The University of Texas M. D. Anderson Cancer Center, and $\ddagger$ Division of Biostatistics, School of Public Health, University of Texas Health Science Center at Houston, Houston, TX; and $\|$ Current address North Coast Cancer Care, Sandusky, OH.

Presented, in part, at the ASCO 2008 Breast Cancer Symposium, September 5-7, 2008, Washington, DC

K.K.H. contributed in conception and design of the work, providing administrative support, data analysis and interpretation, manuscript writing, and gave the final approval of the manuscript. M.Y. contributed in conception and design of the work, collection and assembly of data, data analysis and interpretation, manuscript writing, and gave final approval of the manuscript. S.L. contributed in conception and design of the work, data analysis and interpretation, and gave the final approval of the manuscript. E.H.H., T.A.B., F.M-B., I.B., H.M.K., M.I.R., A.L., B.W.F., W.F.S., A.M.G-A., and A.U.B. contributed in conception and design of the work and gave the final approval of the manuscript.

Reprints: Kelly K. Hunt, MD, Department of Surgical Oncology, Unit 444, The University of Texas M. D. Anderson Cancer Center, 1515 Holcombe Blvd., Houston, TX 77030. E-mail: khunt@mdanderson.org.

Copyright (C) 2011 by Lippincott Williams \& Wilkins

ISSN: 0003-4932/11/25303-0572

DOI: $10.1097 /$ SLA.0b013e318208fc2a
(Ann Surg 2011;253:572-579)

psilateral breast tumor recurrence (IBTR) occurs after breast conservation therapy (BCT) in approximately $8 \%$ to $20 \%$ of patients over a 10 -year period. ${ }^{1-4}$ Optimal management of patients with IBTR after BCT is not well-defined. Numerous reports indicate that IBTR is an independent predictor of distant metastatic disease and poor survival. $^{5-7}$ Others have suggested that there are subgroups of patients who have a relatively favorable prognosis after IBTR ${ }^{8,9}$ One hypothesis is that differences in prognosis among patients with IBTR are because of 2 distinct types of disease: true local recurrences (TR) and new ipsilateral primary tumors (NP). ${ }^{10}$ Another hypothesis is that individuals with NP may have a genetic predisposition for breast carcinoma and are therefore at risk for NP in the treated and contralateral breast. ${ }^{11}$ If these hypotheses are true, clinical management and chemoprevention strategies for patients with IBTR should be tailored to reflect this distinction.

Several studies have attempted to classify 2 distinct types of IBTR by using tumor location, histologic subtype, DNA flow cytometry data, or gene-expression profiling data. ${ }^{11-14}$ In 2002, Huang et al classified IBTR as either NP or TR on the basis of tumor location and histologic subtype and demonstrated that patients with NP had significantly better survival rates than those with TR. ${ }^{11}$ It has been recognized that these classification methods are subject to error (ie, misclassification). ${ }^{11,15}$ Because of the lack of a widely recognized standard for classification, to date the validity of these classification methods has not been evaluated and, thus, most clinicians treat both groups of patients in a similar fashion. Therefore, there is a need to measure the sensitivity and specificity values of the classification methods, as this may be useful to clinicians in developing rational treatment strategies.

In this study, we classified IBTR as either NP or TR using 2 different classification methods. We determined whether those distinctions had prognostic value for patients with IBTR after BCT and also compared the sensitivity and specificity values of the 2 classification methods.

\section{METHODS}

\section{Patient Population}

We reviewed the records of 5660 patients with ductal carcinoma in situ (DCIS) and invasive breast cancer who underwent BCT from 1970 to 2005 at The University of Texas M. D. Anderson Cancer Center. This study was approved by the M. D. Anderson institutional review board.

Ipsilateral breast tumor recurrence was the first site of recurrence in 447 (7.9\%) of patients. A histologically confirmed diagnosis of breast cancer within the previously treated breast was defined as 
an IBTR. Clinical data from 397 (88.8\%) patients were available for review. Of those, 126 had been previously evaluated in the study reported by Huang et al, ${ }^{11}$ and thus, we classified them as the "initial dataset." There were 271 additional patients who were not included in the Huang study, and these patients were classified as the "new dataset."

\section{Study Design}

Using the first classification method (method 1), we classified IBTR as either a NP or TR on the basis of tumor location and histologic subtype. An IBTR was designated TR if it was located within $3 \mathrm{~cm}$ of the primary tumor bed (by breast imaging or clinical examination) and if the histologic subtype was consistent with the primary tumor. If the IBTR was more than $3 \mathrm{~cm}$ from the primary tumor site or had a change in histology, it was designated as NP. For example, a change from infiltrating ductal carcinoma to infiltrating lobular, tubular, or medullary carcinoma would be considered a new primary tumor. We also considered a change from an infiltrating ductal carcinoma to DCIS as characteristic of a NP. We considered a change from DCIS to an infiltrating ductal carcinoma as characteristic of TR.

In the second method (method 2), we classified IBTR as NP or TR on the basis of tumor location, histologic subtype, estrogen receptor (ER) status, and human epidermal growth factor receptor 2 (HER-2) status. Using this method, IBTR was designated TR if it was located within $3 \mathrm{~cm}$ of the primary tumor bed, if the histologic subtype was consistent with the primary tumor, and if both ER and HER-2 status were consistent with the primary tumor. If the IBTR failed to meet any of these 4 criteria, it was designated as NP.

\section{Statistical Analysis}

For statistical analysis, patients in the initial dataset and new dataset were analyzed together and again separately as subgroups, and IBTR within each dataset were classified as TR or NP using the 2 classification methods. Univariate and multivariate logistic regression analyses comparing various clinical and pathologic characteristics between patients with NP and TR were performed for each dataset to determine whether the "new dataset" could validate findings from the "initial dataset". The Kaplan-Meier method and log-rank test were used to calculate and compare actuarial statistics for time to IBTR after BCT and rates of overall and distant disease-free survival.

\section{Calculation of Sensitivity and Specificity Values}

A standard assumption in logistic regression analysis is that the binary outcome is measured perfectly. If the outcome measurement is imperfect and misclassification occurs, the odds ratio estimates will be biased toward the null hypothesis. ${ }^{16}$ In addition, ignoring misclassification errors may cause serious bias in the estimates and power loss in the hypothesis test. ${ }^{17-21}$ It has been shown that corrected odds ratios may be obtained by incorporating external estimates of test sensitivity and specificity into the likelihood for logistic regression. ${ }^{22}$ $\mathrm{Tu}$ et $\mathrm{al}^{23}$ proposed a Bayesian model that incorporates the test sensitivity and specificity into the likelihood function to quantify the effects of covariates on event probability. Their approach has been used in several publications and has the advantages that a relatively broad family of link functions is easily accommodated, data resulting from multiple screening tests and/or pooled samples can be modeled, and the effect of covariates assessed. ${ }^{24,25}$ In this study, we used Tu's method to test the sensitivity and specificity of each method. Even though there is neither a gold standard for the classification method nor validation dataset, the estimates of sensitivity and specificity can still be obtained from the information of 2 sources. First, the covariates, which we used for estimating sensitivity and specificity are strongly associated with the IBTR status. Second, there are 2 methods to classify the same IBTR status, and the additional information from the second method also helps to identify the true IBTR status.

All $P$ values were 2 -tailed, and a value of 0.05 or less was considered significant. R and WinBugs statistical software (R 2.8.1, a GNU project; WinBugs14, The BUGS Project) were used for statistical analyses.

\section{RESULTS}

Analysis was performed with all 397 patients who developed IBTR during the study period. Patient, tumor, and treatment characteristics are summarized in Table 1.

\section{Method 1: Classification of IBTR as NP or TR on the Basis of Tumor Location and Histologic Subtype}

Utilizing method 1 to classify IBTR in the whole dataset, we classified 196 (49.4\%) IBTR as NP and 201 (50.6\%) as TR. We compared clinical and pathological characteristics between patients with IBTR classified as NP and those classified as TR by method 1 (Table 2). Patients classified as TR were younger when they had IBTR compared with patients classified as NP (TR 52.9 years old vs. NP 55.9 years old; $P=0.03$ ). Patients classified as TR were more likely to have chemotherapy for IBTR compared with patients classified as NP (TR 51.2\% vs. NP $38.3 \% ; P=0.009$ ). Patients classified as NP were more likely to have surgery for IBTR tumor compared with patients classified as TR (NP 96.9\% vs. TR $89.5 \% ; P=0.006$ ). Patients classified as TR had a much higher rate of systemic recurrence after IBTR (TR $42.3 \%$ vs. NP $10.7 \% ; P<.0001$ ). Patients classified as NP had a significantly higher rate of contralateral breast carcinoma (TR $9.5 \%$ vs. NP $16.3 \% ; P=0.04)$. The time between BCT and IBTR was shorter for patients classified as TR than for those classified as NP (mean, TR 5.4 years vs. NP 7.6 years; $P<0.0001$, log-rank test). No other significant differences in patient characteristics were noted.

Actuarial survival rates showed that patients with IBTR classified as NP had more favorable outcomes (Fig. 1). Figure 1 shows that overall (Fig. 1a) and disease-free survival rates (Fig. 1c) from the date of recurrence for patients classified as NP were significantly better than those for patients classified as TR.

In the univariate and multivariate analyses, clinical T-stage of the primary, age at time of recurrence, skin or lymph node involvement at IBTR, and recurrence type classified by method 1 significantly affected disease-free survival (Table 3). Patients with higher clinical T-stage, IBTR classified as TR, younger age at recurrence, or skin or lymph node involvement at IBTR had worse disease-free survival. We also analyzed whether local and systemic treatments for IBTR had an impact on the disease-free survival of the IBTR groups (Fig. 2). Figure $2 \mathrm{a}$ shows that disease-free survival rate for patients classified as TR treated with chemotherapy for IBTR were significantly worse than others (TR without chemotherapy, NP treated with chemotherapy, NP without chemotherapy). Figure $2 b$ shows that disease-free survival rates for patients classified as TR not treated with surgery for IBTR were significantly worse than others (TR treated with surgery, $\mathrm{NP}$ ). Whether patients had segmental mastectomy or total mastectomy for their IBTR did not seem to have an impact on survival rates. Whether patients had hormonal therapy for treatment of the IBTR also did not seem to have an impact on survival rates.

We also performed subgroup analyses using the initial dataset describing the classification of NP and TR and the new dataset with patients from a more contemporary treatment period. We found similar results in both datasets compared with the analysis of the whole dataset. 
TABLE 1. Clinical and Pathologic Characteristics of 397

Patients With Ipsilateral Breast Tumor Recurrence After Breast-Conserving Therapy

\begin{tabular}{|c|c|}
\hline Characteristic & Percent (\%) \\
\hline \multicolumn{2}{|l|}{ Age, $y$} \\
\hline At primary diagnosis, mean (range) & $47.5(23-85)$ \\
\hline At IBTR, mean (range) & $54.2(29-90)$ \\
\hline Time to IBTR in years, mean (range) & $6.5(0.1-24.5)$ \\
\hline Follow-up time in years, mean (range) & $12.2(1.3-34.3)$ \\
\hline \multicolumn{2}{|l|}{ Location of primary tumor } \\
\hline Upper inner quadrant & 13.0 \\
\hline Upper outer quadrant & 43.5 \\
\hline Lower inner quadrant & 10.4 \\
\hline Lower outer quadrant & 8.5 \\
\hline Central & 24.6 \\
\hline \multicolumn{2}{|l|}{ Contralateral breast cancer } \\
\hline Yes & 12.9 \\
\hline No & 87.1 \\
\hline \multicolumn{2}{|l|}{ Radiation for primary tumor } \\
\hline Yes & 84.1 \\
\hline No & 15.9 \\
\hline \multicolumn{2}{|l|}{ Adjuvant chemotherapy for primary tumor } \\
\hline Yes & 30.2 \\
\hline No & 69.8 \\
\hline \multicolumn{2}{|c|}{ Adjuvant hormonal therapy for primary tumor } \\
\hline Yes & 22.2 \\
\hline No & 77.8 \\
\hline \multicolumn{2}{|l|}{ Clinical T stage of primary tumor } \\
\hline Tis/T1 & 72.0 \\
\hline $\mathrm{T} 2 / \mathrm{T} 3$ & 28.0 \\
\hline \multicolumn{2}{|l|}{ Histology of primary tumor } \\
\hline Invasive ductal carcinoma & 64.0 \\
\hline Invasive lobular carcinoma & 4.0 \\
\hline Mixed ductal and lobular carcinoma & 3.0 \\
\hline Ductal carcinoma in situ & 20.9 \\
\hline Other & 8.1 \\
\hline \multicolumn{2}{|l|}{ Lymph node involvement } \\
\hline Primary tumor & 18.6 \\
\hline IBTR & 15.6 \\
\hline \multicolumn{2}{|l|}{ Nuclear grade of primary tumor } \\
\hline I & 7.9 \\
\hline II & 44.9 \\
\hline III & 47.2 \\
\hline \multicolumn{2}{|l|}{ Margin of primary tumor } \\
\hline Positive & 8.6 \\
\hline Negative & 86.6 \\
\hline Unknown & 4.8 \\
\hline \multicolumn{2}{|l|}{ Systemic recurrence } \\
\hline No & 73.3 \\
\hline Yes & 26.7 \\
\hline \multicolumn{2}{|l|}{ Skin involvement at IBTR } \\
\hline No & 83.1 \\
\hline Yes & 13.1 \\
\hline Unknown & 3.8 \\
\hline
\end{tabular}

TABLE 1. (Continued)

\begin{tabular}{lc} 
Characteristic & Percent (\%) \\
\hline Positive & 60.3 \\
Negative & 39.7 \\
PR status of primary tumor & \\
Positive & 54.7 \\
Negative & 45.3 \\
HER-2 status of primary tumor & \\
Positive & 24.8 \\
Negative & 75.2 \\
\hline \multicolumn{2}{l}{ PR indicates progesterone receptor. } \\
\hline
\end{tabular}

\section{Method 2: Classification of IBTR as NP or TR on the Basis of Tumor Location, Histologic Subtype, and ER and HER-2 Status}

Utilizing method 2 to classify IBTR with the whole dataset, we classified $212(53.4 \%)$ IBTR as NP and $185(46.6 \%)$ as TR. After comparing clinical and pathological characteristics between patients with IBTR classified as NP and those classified as TR by method 2 (Table 2), we found some similarities with method 1: a higher rate of contralateral breast carcinoma in patients classified as NP; a higher rate of systemic recurrence after IBTR in patients classified as TR; patients classified as TR were more likely to have chemotherapy for IBTR; patients classified as TR were less likely to have surgery for IBTR. We also noted that the time between BCT and IBTR was shorter for patients classified as TR as compared with those classified as NP (mean, TR 5.4 years vs. NP 7.4 years; $P=0.0003, \log$-rank test). In contrast to method 1 , there was no significant difference in patient age at IBTR between the 2 groups using the method 2 classification.

Actuarial survival rates show that patients with IBTR classified as NP had more favorable outcomes. Figure 1 shows that overall (Fig. 1b) and disease-free survival (Fig. 1d) rates for all patients classified as NP were significantly better than those of patients classified as TR by method 2 .

In the univariate and multivariate analyses, clinical T-stage of the primary, age at the time of IBTR, skin or lymph node involvement at IBTR, and IBTR type classified by method 2 significantly affected disease-free survival (Table 3). Patients with higher clinical T-stage, IBTR classified as TR, younger age at recurrence, or skin or lymph node involvement at IBTR had worse disease-free survival.

Subgroup analyses were done using the initial and the new datasets and we found similar results in both datasets compared with the whole dataset.

\section{Sensitivity and Specificity}

Multivariate analysis, sensitivity and specificity are shown in Table 4. In multivariate analysis, we found that patients classified as TR had a much higher rate of systemic recurrence after IBTR; patients classified as NP still had a significantly higher rate of contralateral breast carcinoma; and patients classified as TR had shorter recurrence time in both method 1 and method 2 . The sensitivity and specificity values for method 1 were 0.812 and 0.867 , respectively. The sensitivity and specificity values for method 2 were 0.870 and 0.800 , respectively.

\section{DISCUSSION}

In this study, we used 2 different methods to classify IBTR as NP or TR and demonstrated that both easily applicable methods had significant prognostic value. Using both classification methods, 
TABLE 2. Comparison of Clinical, Pathological, and Treatment Characteristics Between Patients With NP and TR

\begin{tabular}{|c|c|c|c|c|c|c|}
\hline \multirow[b]{2}{*}{ Characteristics } & \multicolumn{3}{|c|}{ Method 1} & \multicolumn{3}{|c|}{ Method 2} \\
\hline & $\operatorname{TR} n=201(\%)$ & NP $n=196(\%)$ & $P$ & TR $n=185(\%)$ & $N P n=212(\%)$ & $\boldsymbol{P}$ \\
\hline \multicolumn{7}{|l|}{ Age, y (mean) } \\
\hline At primary & 47.4 & 47.6 & 0.79 & 47.5 & 47.5 & 0.95 \\
\hline At IBTR & 52.9 & 55.9 & 0.03 & 53.1 & 55.1 & 0.08 \\
\hline Time to IBTR in years & 5.4 & 7.6 & $<0.0001^{*}$ & 5.5 & 7.4 & $0.0001^{*}$ \\
\hline Follow-up time in years & 10.8 & 13.4 & 0.0006 & 11.0 & 13.1 & 0.007 \\
\hline Contralateral breast cancer & & & 0.04 & & & 0.02 \\
\hline Yes & $19(9.4)$ & $32(16.3)$ & & $16(8.7)$ & $35(16.5)$ & \\
\hline No & $182(90.6)$ & $164(86.7)$ & & $169(91.3)$ & $177(83.5)$ & \\
\hline Radiation for primary tumor & & & 0.81 & & & 0.36 \\
\hline Yes & $170(84.6)$ & $164(83.7)$ & & $159(86.0)$ & $175(82.6)$ & \\
\hline No & $31(15.4)$ & $32(16.3)$ & & $26(14.0)$ & $37(17.4)$ & \\
\hline Adjuvant chemotherapy for primary tumor & & & 0.41 & & & 0.39 \\
\hline Yes & $57(28.4)$ & $63(32.1)$ & & $52(28.1)$ & $68(32.1)$ & \\
\hline No & $144(71.6)$ & $133(67.9)$ & & $133(71.9)$ & $144(67.9)$ & \\
\hline Adjuvant hormonal therapy for primary tumor & & & 0.18 & & & 0.33 \\
\hline Yes & $39(19.4)$ & $49(25.0)$ & & $37(20.0)$ & $51(24.1)$ & \\
\hline No & $162(80.6)$ & $147(75.0)$ & & $148(80.0)$ & $161(75.9)$ & \\
\hline ER status of primary & & & $0.19 \dagger$ & & & $0.81 \dagger$ \\
\hline Positive & $86(56.6)$ & $99(63.9)$ & & $83(61.0)$ & $102(59.7)$ & \\
\hline Negative & $66(43.4)$ & $56(36.1)$ & & $53(39.0)$ & $69(40.3)$ & \\
\hline PR status of primary & & & $0.29 \dagger$ & & & $0.96 \dagger$ \\
\hline Positive & $71(51.4)$ & $86(57.7)$ & & $68(54.8)$ & $89(54.6)$ & \\
\hline Negative & $67(48.6)$ & $63(42.3)$ & & $56(45.2)$ & $74(45.4)$ & \\
\hline HER-2 status of primary & & & $0.88 \dagger$ & & & $0.97 \dagger$ \\
\hline Positive & $17(25.4)$ & $17(24.3)$ & & $14(25.0)$ & $20(24.7)$ & \\
\hline Negative & $50(74.6)$ & $53(75.7)$ & & $42(75.0)$ & $61(75.3)$ & \\
\hline Chemotherapy for IBTR & & & 0.009 & & & 0.04 \\
\hline Yes & $103(51.2)$ & $75(38.3)$ & & $93(20.3)$ & $85(40.1)$ & \\
\hline No & $98(48.8)$ & $121(61.7)$ & & $92(49.7)$ & $127(59.9)$ & \\
\hline Hormonal therapy for IBTR & & & 0.58 & & & 0.65 \\
\hline Yes & $72(35.8)$ & $65(33.2)$ & & $66(35.7)$ & $71(33.5)$ & \\
\hline No & $129(64.2)$ & $131(66.8)$ & & $119(64.3)$ & $141(66.5)$ & \\
\hline ER status of IBTR & & & $0.93 \dagger$ & & & $0.65 \dagger$ \\
\hline Positive & $102(64.2)$ & $106(64.6)$ & & $94(65.7)$ & $114(63.3)$ & \\
\hline Negative & $57(35.8)$ & $58(35.4)$ & & $49(32.3)$ & $66(36.7)$ & \\
\hline PR status of IBTR & & & $0.31 \dagger$ & & & $0.26 \dagger$ \\
\hline Positive & $81(56.3)$ & $73(50.3)$ & & $74(56.9)$ & $80(50.3)$ & \\
\hline Negative & $63(43.7)$ & $72(50.0)$ & & $56(43.1)$ & $79(49.7)$ & \\
\hline HER-2 status of IBTR & & & $0.48 \dagger$ & & & $0.13 \dagger$ \\
\hline Positive & $17(19.1)$ & $24(23.3)$ & & $12(15.8)$ & $29(25.0)$ & \\
\hline Negative & $72(80.9)$ & $79(76.7)$ & & $64(84.2)$ & $87(75.0)$ & \\
\hline Surgery for IBTR & & & 0.006 & & & 0.01 \\
\hline No surgery & $21(10.5)$ & $6(3.1)$ & & $20(10.8)$ & $7(3.3)$ & \\
\hline SM & $30(14.9)$ & $23(11.7)$ & & $24(13.0)$ & $29(13.7)$ & \\
\hline $\mathrm{TM}$ & $150(74.6)$ & $167(85.2)$ & & $141(76.2)$ & $176(83.0)$ & \\
\hline Systemic recurrence & & & $<0.0001$ & & & $<0.0001$ \\
\hline Yes & $85(42.3)$ & $21(10.7)$ & & $78(42.2)$ & $28(13.2)$ & \\
\hline No & $116(57.7)$ & $175(89.3)$ & & $107(57.8)$ & $184(86.8)$ & \\
\hline
\end{tabular}



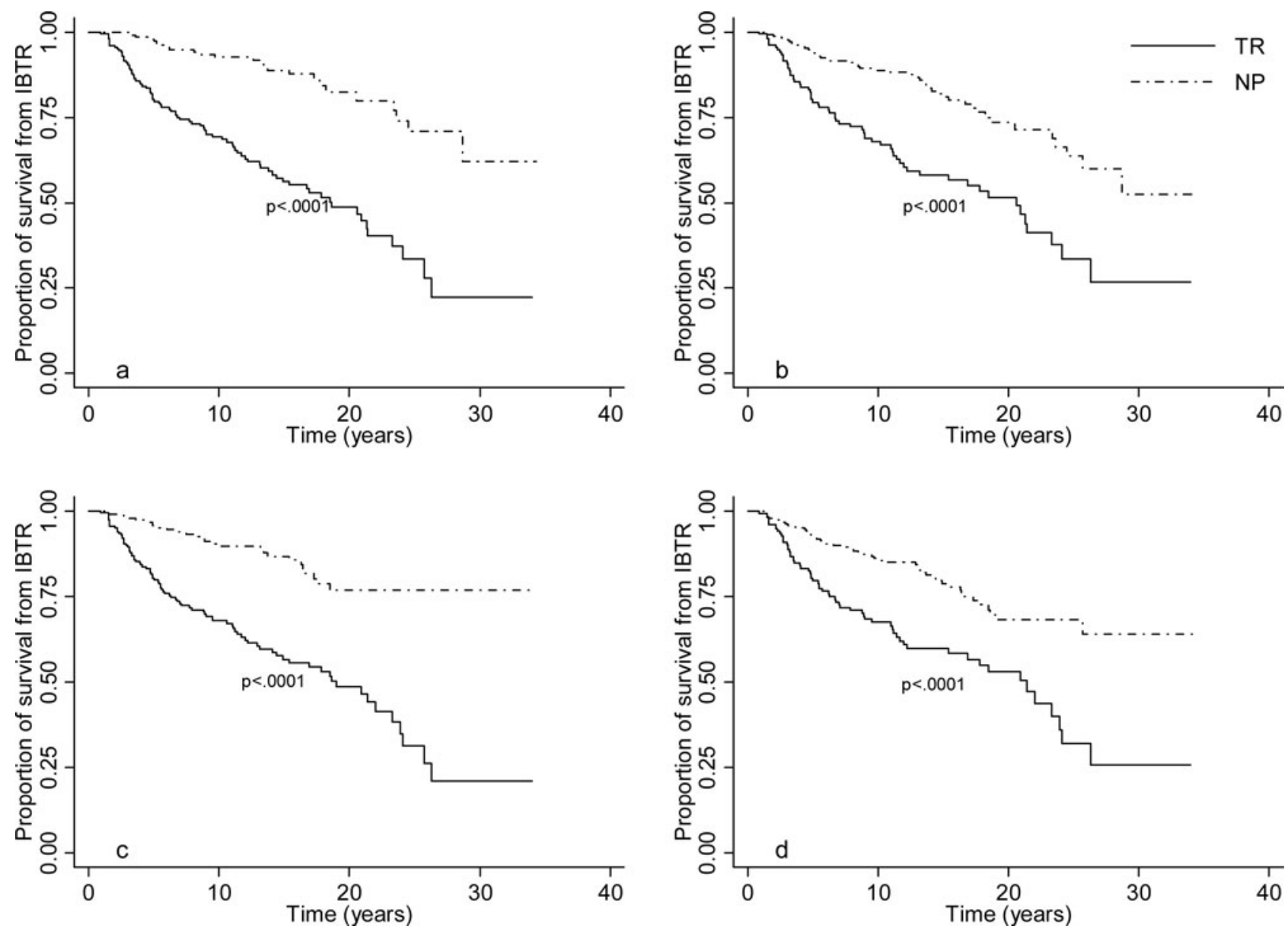

FIGURE 1. Actuarial survival curves using data from all 397 patients with IBTR showing improved survival times from the time of first cancer treatment for patients classified as having a new primary tumor (NP) compared with patients with true local disease recurrence (TR). Curves calculated with data from either the a, overall survival by method 1; b, overall survival by method 2; $c$, disease-free survival by method 1 ; or $d$, disease-free survival by method 2 .

TABLE 3. Univariate and Multivariate Analyses for Systemic Disease-Free Survival by Method for the Combined Dataset $(n=397)$

\begin{tabular}{|c|c|c|c|c|c|c|}
\hline \multirow[b]{2}{*}{ Characteristics } & \multirow[b]{2}{*}{$\begin{array}{c}\text { Univariate } \\
\text { (HR 95\% CI) }\end{array}$} & \multirow[b]{2}{*}{$\boldsymbol{P}$} & \multicolumn{2}{|c|}{ Method 1} & \multicolumn{2}{|c|}{ Method 2} \\
\hline & & & $\begin{array}{l}\text { Multivariate } \\
\text { HR (95\% CI) }\end{array}$ & $\begin{array}{c}\text { Multivariate } \\
\boldsymbol{P}\end{array}$ & $\begin{array}{c}\text { Multivariate } \\
\text { HR (95\% CI) }\end{array}$ & $\begin{array}{c}\text { Multivariate } \\
P\end{array}$ \\
\hline Age at recurrence & $0.9(0.95-0.98)$ & $<0.0001$ & $0.98(0.96-0.99)$ & 0.018 & $0.98(0.96-0.99)$ & 0.016 \\
\hline \multicolumn{7}{|l|}{ Clinical T-stage of primary tumor } \\
\hline $\mathrm{T} 0$ or $\mathrm{T} 1$ & Referent & & & & & \\
\hline $\mathrm{T} 2$ or $\mathrm{T} 3$ & $2.3(1.6-3.3)$ & $<0.0001$ & $2.1(1.4-3.1)$ & $<.0001$ & $2.2(1.5-3.2)$ & $<0.0001$ \\
\hline \multicolumn{7}{|l|}{ Recurrence type } \\
\hline True recurrence & Referent & & Referent & & Referent & \\
\hline New primary tumor & $0.4(0.3-0.6)$ & $<0.0001$ & $0.3(0.2-0.4)$ & $<.0001$ & $0.3(0.2-0.5)$ & $<0.0001$ \\
\hline Skin involvement at IBTR & $4.5(3.0-6.8)$ & $<0.0001$ & $1.8(1.4-2.5)$ & $<.0001$ & $1.8(1.4-2.4)$ & $<0.0001$ \\
\hline Lymph node involvement at IBTR & $3.6(2.4-5.4)$ & $<0.0001$ & $2.3(1.5-3.4)$ & $<.0001$ & $2.4(1.6-3.6)$ & $<0.0001$ \\
\hline
\end{tabular}



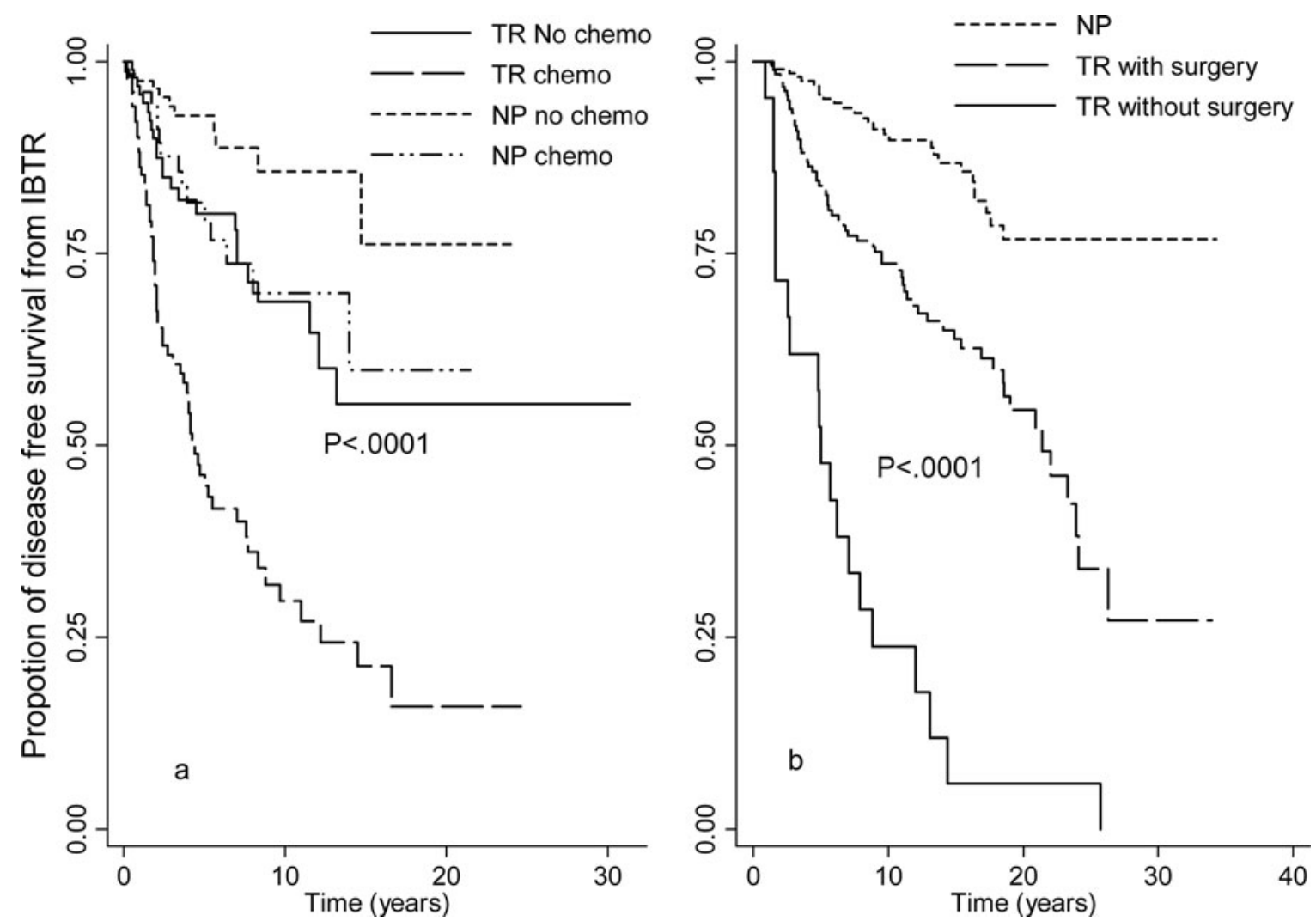

FIGURE 2. Actuarial survival curves using data from all 397 patients with IBTR showing improved survival times from the time of first cancer treatment for patients classified as having a new primary tumor (NP) compared with patients with true local disease recurrence (TR) under different treatments for the recurrent tumor. a, Whether patients had chemotherapy for recurrent tumor in NP and TR groups; b, whether patients had surgery for recurrent tumor in NP and TR groups.

patients classified as NP had more favorable overall and disease-free survival rates than those classified as TR.

Our findings support the results of other studies that have attempted to define indicators of prognosis after IBTR. Specifically, the features of NPs have been correlated with better outcomes; including longer time from BCT to IBTR and recurrence at a location of more than $3 \mathrm{~cm}$ from the primary tumor site. Our data also support the hypothesis that a NP is a disease entity independent from the primary breast carcinoma. The subgroup of patients classified as NP had much better outcomes than those classified as TR. This observation makes intuitive sense because patients with a NP should have a prognosis similar to patients with new early-stage primary breast carcinomas.

Given the fact that NP- and TR-IBTRs have different natural histories and different prognoses, the clinical implications for their therapeutic management may also differ. Patients with TR have poorer prognoses both in terms of survival rates and the development of distant tumor recurrence, and may benefit from more aggressive treatments. ${ }^{26}$ Our study demonstrates that even with more aggressive treatments, patients classified as TR still had worse survival rates compared with patients classified as NP. Despite the increased utilization of systemic treatment, patients with TR were more likely to develop distant metastasis and death due to breast cancer. Many of these patients did not undergo surgery for their IBTR, likely because of detection of distant metastasis. This suggests that patients classified as TR are more likely to harbor distant metastatic disease at the time of IBTR and may be candidates for novel systemic treatment strategies. We have shown that patients with NPs generally have favorable long-term prognoses. Decisions concerning systemic therapy for these patients should be similar to those for patients with equivalent-stage first primary breast carcinomas.

The decision rules for classification of IBTR differ among previous studies. The location of tumor recurrence is a significant part of the determination of classification in most studies. Many reports have based the judgment regarding TR versus NP on the difference in location between the initial tumor and the subsequent tumor in the treated breast. ${ }^{2,7,27}$ In addition to location, some studies have considered histological subtype to distinguish TR from NP. ${ }^{11,12,28}$ Pathological findings that have been proposed are the presence of previously unidentified carcinoma in situ and better differentiation of the tumor (infiltrating to noninfiltrating changes and high-grade to low-grade changes) ${ }^{29,30}$ DNA flow cytometry findings have also been included as criteria. ${ }^{14,30}$ Recently, allelic imbalances or loss of heterozygosity detected by DNA fingerprinting showed that these new evaluation methods could predict the patient's outcome more precisely than conventional methods. ${ }^{15}$ Some reports suggest that the tumor marker status is unchanged from the primary when an IBTR is a TR. ${ }^{31,32}$ Others have reported loss of hormone receptors in recurrent tumors correlating with a poor response to endocrine therapy. ${ }^{33-35}$ Recent studies have shown differences between primary and residual breast cancer after chemotherapy, ${ }^{36}$ and primary and recurrent or metastatic disease. ${ }^{37,38}$ To provide a clinically useful method that is readily available to most clinicians, we used 2 different methods to classify IBTR as TR or NP. Both methods showed that IBTR can be 
TABLE 4. Comparison of the Odds Ratios and the Confidence Intervals of Clinicopathologic Factors and Occurrence of NP Compared to TR Classified by Method 1 and Method 2 Using Different Statistical Approaches

\begin{tabular}{|c|c|c|c|c|}
\hline \multirow[b]{2}{*}{ Covariates } & \multicolumn{2}{|c|}{ Logistic Regression } & \multicolumn{2}{|c|}{ Tu's Method } \\
\hline & Method 1 & Method 2 & Method 1 & Method 2 \\
\hline Number of new primary & $165(53.2 \%)$ & $213(53.7 \%)$ & & \\
\hline Contralateral breast cancer & $0.69 *(0.02-1.36) \dagger$ & $0.80 *(0.12-1.49) \dagger$ & $1.28 *(0.21-3.22) \dagger$ & $1.50 *(0.43-3.52)$ \\
\hline Time to IBTR & $0.06^{*}(0.02-0.10) \dagger$ & $0.05^{*}(0.01-0.09) \dagger$ & $0.118^{*}(0.04-0.31) \dagger$ & $0.08^{*}(0.02-0.18) \dagger$ \\
\hline No systemic recurrence & $-1.74 *(-2.28$ to -1.19$) \dagger$ & $-1.51^{*}(-2.01$ to -1.00$) \dagger$ & $-3.99 *(-9.55$ to -1.84$) \dagger$ & $-4.04^{*}(-9.67$ to -1.63$) \dagger$ \\
\hline Sensitivity & $1.0^{*}$ (Assumed) & $1.0^{*}$ (Assumed) & $0.820^{*}(0.663-0.986) \dagger$ & $0.870^{*}(0.716-0.993) \dagger$ \\
\hline Specificity & $1.0^{*}$ (Assumed) & $1.0^{*}$ (Assumed) & $0.867 *(0.763-0.989) \dagger$ & $0.800 *(0.690-0.977) \dagger$ \\
\hline
\end{tabular}

${ }^{*}$ Estimated Coefficient; $\uparrow 95 \%$ confidence interval

classified as TR or NP and that these classifications have different natural histories and different prognoses.

Because of the lack of a widely recognized gold standard for classification of IBTRs, there was no direct method for determining which of the 2 methods was better at classifying IBTR as NP or TR. We used Tu's method ${ }^{23}$ to obtain estimated sensitivity and specificity values for each method. Method 2 had higher sensitivity but lower specificity than method 1 . If we assume that NP represents disease and TR represents nondisease in the conventional definitions of sensitivity and specificity, the increased sensitivity is likely because method 2 uses 2 more criteria than method 1. Our study provides accurate estimates of sensitivity and specificity for each classification method to help clinicians decide which method to use based on the sensitivity-specificity trade-off. Clinicians may choose method 2 if higher sensitivity is preferred and method 1 if higher specificity is preferred. The difference between the methods is the ability to add tumor markers (ER, HER-2) in the contemporary dataset, which may further discriminate NP from TR. As the number of patients with complete tumor markers from both the initial diagnosis and IBTR was limited, we did not see a significant difference between the 2 classification methods. In the future when the tumor markers are more routinely performed and available on both the primary tumor and IBTR, method 2 may be more relevant.

Recently, molecular classifications of invasive carcinoma have been assessed in predicting IBTR. The 21-gene recurrence score (RS) assay, which was developed to predict distant failure in patients with ER-positive cancers treated with tamoxifen, ${ }^{39,40}$ has also been shown to predict risk of local recurrence. ${ }^{41}$ Mamounas et $\mathrm{al}^{41}$ found that RS assay was a significant predictor of local-regional recurrence in tamoxifen-treated patients. Millar et $\mathrm{al}^{42}$ applied a 5-biomarker panel to predict local, distant recurrence and death after $\mathrm{BCT}$, and found median time to event for all measures of outcome with greatly shortened recurrence times for the more aggressive subtypes: basal, HER-2, and unclassified. ${ }^{42}$ Using the 21 -gene RS assay or a 5-biomarker panel has not yet been tested in terms of classifying IBTR to TR or NP; however, they do provide additional information to clinicians regarding expected outcomes after BCT, and the more aggressive subtypes identified with these methods may in fact represent TR.

The main limitations of this study are that IBTRs were classified using only clinical and pathological criteria, without molecular confirmation and that an assumption was made that molecular markers are fixed between the primary tumor and TR. This assumption may not always be true because markers may change because of clonal evolution. Although Tu's method ${ }^{23}$ provides the corrected coefficient estimates and allows for uncertainty in test sensitivity and specificity values, the estimates can be further improved by incorporating additional survival information that is correlated with the classification status. In the future, working with statisticians to develop a methodological framework that provides more accurate estimates of sensitivity and specificity values for each classification method will help clinicians decide which method to use on the basis of the sensitivity-specificity trade-off. More precise molecular studies will likely be able to identify the clonal relatedness of the IBTR and the primary tumor. Our methodology, based on readily available information, has greater clinical applicability than molecular techniques that require sophisticated laboratory analyses. Our results demonstrate that these criteria can identify subgroups of patients with significantly different outcomes after IBTR.

In conclusion, TR and NP show quite different features. This suggests that classifying IBTR as TR or NP using the methods described here can provide clinically significant data for the management of IBTR both systemically and locally.

\section{REFERENCES}

1. Chen AM, Meric-Bernstam F, Hunt KK, et al. Breast conservation after neoadjuvant chemotherapy: the MD Anderson cancer center experience. J Clin Oncol. 2004;22(12):2303-2312.

2. Fowble B, Solin LJ, Schultz DJ, et al. Breast recurrence following conservative surgery and radiation: patterns of failure, prognosis, and pathologic findings from mastectomy specimens with implications for treatment. Int $J$ Radiat Oncol Biol Phys. 1990;19(4):833-842.

3. Rouzier R, Extra JM, Carton M, et al. Primary chemotherapy for operable breast cancer: incidence and prognostic significance of ipsilateral breast tumor recurrence after breast-conserving surgery. J Clin Oncol. 2001;19(18): 3828-3835.

4. van Dongen JA, Voogd AC, Fentiman IS, et al. Long-term results of a randomized trial comparing breast-conserving therapy with mastectomy: European Organization for Research and Treatment of Cancer 10801 trial. J Natl Cancer Inst. 2000;92(14):1143-1150.

5. Fisher B, Anderson S, Fisher ER, et al. Significance of ipsilateral breast tumour recurrence after lumpectomy. Lancet. 1991;338(8763):327-331.

6. Haffty BG, Fischer D, Beinfield M, et al. Prognosis following local recurrence in the conservatively treated breast cancer patient. Int J Radiat Oncol Biol Phys. 1991;21(2):293-298.

7. Kurtz JM, Amalric R, Brandone H, et al. Local recurrence after breastconserving surgery and radiotherapy. Frequency, time course, and prognosis. Cancer. 1989;63(10):1912-1917.

8. Anderson SJ, Wapnir I, Dignam JJ, et al. Prognosis after ipsilateral breast tumor recurrence and locoregional recurrences in patients treated by breastconserving therapy in five national surgical adjuvant breast and bowel project protocols of node-negative breast cancer. J Clin Oncol. 2009;27(15):24662473.

9. Wapnir IL, Anderson SJ, Mamounas EP, et al. Prognosis after ipsilateral breast tumor recurrence and locoregional recurrences in five National Surgical Adjuvant Breast and Bowel Project node-positive adjuvant breast cancer trials. $J$ Clin Oncol. 2006;24(13):2028-2037. 
10. Veronesi U, Marubini E, Del Vecchio M, et al. Local recurrences and distant metastases after conservative breast cancer treatments: partly independent events. J Natl Cancer Inst. 1995;87(1):19-27.

11. Huang E, Buchholz TA, Meric F, et al. Classifying local disease recurrences after breast conservation therapy based on location and histology: new primary tumors have more favorable outcomes than true local disease recurrences. Cancer. 2002;95:2059-2067.

12. Abd-Alla HM, Lotayef MM, Abou Bakr A, et al. Ipsilateral in-breast tumor relapse after breast conservation therapy: true recurrence versus new primary tumor. J Egypt Natl Canc Inst. 2006;18(3):183-190.

13. Nimeus-Malmstrom E, Krogh M, Malmstrom P, et al. Gene expression profiling in primary breast cancer distinguishes patients developing local recurrence after breast-conservation surgery, with or without postoperative radiotherapy. Breast Cancer Res. 2008;10(2):R34.

14. Smith TE, Lee D, Turner BC, et al. True recurrence vs. new primary ipsilateral breast tumor relapse: an analysis of clinical and pathologic differences and their implications in natural history, prognoses, and therapeutic management. Int J Radiat Oncol Biol Phys. 2000;48:1281-1289.

15. Schlechter BL, Yang Q, Larson PS, et al. Quantitative DNA fingerprinting may distinguish new primary breast cancer from disease recurrence. $J$ Clin Oncol. 2004;22:1830-1838.

16. Copeland KT, Checkoway H, McMichael AJ, et al. Bias due to misclassification in the estimation of relative risk. Am J Epidemiol. 1977;105(5): 488-495.

17. Johnson WO, Gastwirth JL. Bayesian Inference for medical screening tests: approximations useful for the analysis of acquired immune deficiency syndrome. J Royal Stat Soc, SerB: Methodol. 1991;53:427-439.

18. Mendoza-Blanco JR, Tu XM, Iyengar S. Bayesian inference on prevalence using a missing-data approach with simulation-based techniques: applications to HIV screening. Stat Med. 1996;15:2161-2176.

19. Rogan WJ, Gladen B. Estimating prevalence from the results of a screening test. Am J Epidemiol. 1978;107(1):71-76.

20. Tu XM, Litvak E, Pagano M. Issues in HIV screening programs. Am J Epidemiol. 1992;136:244-255.

21. Tu XM, Litvak E, Pagano M. On the informativeness and accuracy of pooled testing in estimating prevalence of a rare disease: application to HIV screening. Biometrika. 1995;82:287-297.

22. Magder LS, Hughes JP. Logistic regression when the outcome is measured with uncertainty. Am J Epidemiol. 1997;146(2):195-203.

23. Tu XM, Kowalski J, Jia G. Bayesian analysis of prevalence with covariates using simulation-based techniques: applications to HIV Screening. Stat Med. 1999;18:3059-3073.

24. McInturff P, Johnson WO, Cowling D, et al. Modelling risk when binary outcomes are subject to error. Stat Med. 2004;23(7):1095-1109.

25. Paulino CD, Soares P, Neuhaus J. Binomial regression with misclassification. Biometrics. 2003;59(3):670-675.

26. Haffty BG, Reiss M, Beinfield M, et al. Ipsilateral breast tumor recurrence as a predictor of distant disease: implications for systemic therapy at the time of local relapse. J Clin Oncol. 1996;14:52-57.
27. Recht A, Silen W, Schnitt SJ, et al. Time-course of local recurrence following conservative surgery and radiotherapy for early stage breast cancer. Int J Radiat Oncol Biol Phys. 1988;15(2):255-261.

28. Komoike Y, Akiyama F, Iino Y, et al. Analysis of ipsilateral breast tumor recurrences after breast-conserving treatment based on the classification of true recurrences and new primary tumors. Breast Cancer. 2005;12:104-111.

29. Chaudary MA, Millis RR, Hoskins EO, et al. Bilateral primary breast cancer: a prospective study of disease incidence. Br J Surg. 1984;71(9):711-714.

30. Haffty BG, Carter D, Flynn SD, et al. Local recurrence versus new primary: clinical analysis of 82 breast relapses and potential applications for genetic fingerprinting. Int J Radiat Oncol Biol Phys. 1993;27:575-583.

31. Iglehart JD, Kerns BJ, Huper G, et al. Maintenance of DNA content and erbB2 alterations in intraductal and invasive phases of mammary cancer. Breast Cancer Res Treat. 1995;34(3):253-263.

32. Lacroix H, Iglehart JD, Skinner MA, et al. Overexpression of erbB-2 or EGF receptor proteins present in early stage mammary carcinoma is detected simultaneously in matched primary tumors and regional metastases. Oncogene. 1989;4(2):145-151.

33. Kuukasjarvi T, Kononen J, Helin H, et al. Loss of estrogen receptor in recurrent breast cancer is associated with poor response to endocrine therapy. $J$ Clin Oncol. 1996;14(9):2584-2589.

34. Osborne CK. Heterogeneity in hormone receptor status in primary and metastatic breast cancer. Semin Oncol. 1985;12(3):317-326.

35. Shimizu C, Fukutomi T, Tsuda $\mathrm{H}$, et al. c-erbB-2 protein overexpression and p53 immunoreaction in primary and recurrent breast cancer tissues. J Surg Oncol. 2000;73(1):17-20.

36. Mittendorf EA, Wu Y, Scaltriti M, et al. Loss of her2 amplification following trastuzumab-based neoadjuvant systemic therapy and survival outcomes. Clin Can Res. 2009;15(23):7381-7388.

37. Akcakanat A, Sahin A, Shaye AN, et al. Comparison of Akt/mTOR signaling in primary breast tumors and matched distant metastases. Cancer. 2008;112(11):2352-2358.

38. Liedtke $\mathrm{C}$, Broglio K, Moulder S, et al. Prognostic impact of discordance between triple-receptor measurements in primary and recurrent breast cancer. Ann Oncol. 2009;20(12):1953-1958.

39. Habel LA, Shak S, Jacobs MK, et al. A population-based study of tumor gene expression and risk of breast cancer death among lymph node-negative patients. Breast Cancer Res. 2006;8(3):R25.

40. Paik S, Shak S, Tang G, et al. A multigene assay to predict recurrence of tamoxifen-treated, node-negative breast cancer. $N$ Engl $\mathrm{J} \mathrm{Med}$ 2004;351(27):2817-2826.

41. Mamounas E, Tang G, Bryant J, et al. Association between the 21-gene recurrence score assay (RS) and risk of locoregional failure in node-negative, ER-positive breast cancer: results from NSABP B-14 and NSABP B-20. Breast Cancer Res Treat. 2005;94(suppl.1):S16.

42. Millar EK, Graham PH, O'Toole SA, et al. Prediction of local recurrence, distant metastases, and death after breast-conserving therapy in earlystage invasive breast cancer using a five-biomarker panel. J Clin Oncol. 2009;27(28):4701-4708. 